\title{
Design of Predistortion Linearizer for GaN Power Amplifier
}

\author{
Wang Chong $^{\mathrm{a} *}$ \\ The 54th Research institute of CETC, Shijiazhuang Hebei 050081, China \\ a1462576604@qq.com
}

Keywords: GaN; solid-state power amplifier; open loop; analog predistortion; Schottky diode.

\begin{abstract}
A linearizer using analog pre-distortion is proposed in this paper to improve the non-linear distortion of $\mathrm{GaN}$ power amplifier at $29 \mathrm{GHz} 31 \mathrm{GHz}$. It is improved on the basis of traditional predistorter and composed of two parallel Schottky diodes using open loop method. It can generate the distorted signals which are on different degree of improvement through changing the offset state of the diode. The initial value can be gained by using the ADS2013 which is a specialized electromagnetic simulation software. Then the value gained provides the theoretical basis for physical object debug. The physical object tests show that the linearizer can provide $6.4 \mathrm{~dB}$ gain expansion and $28^{\circ}$ phase expansion at $30 \mathrm{GHz}$.
\end{abstract}

\section{Introduction}

The third generation of semiconductor materials $\mathrm{GaN}$ has been applied to the power amplifier, at the same time, in order to meet the higher speed transmission of large capacity data, Orthogonal frequency division multiplexing (OFDM) and quadrature amplitude modulation techniques (such as 16QAM, 64QAM, etc.) are widely used, which puts a higher demand on the linearity of the power amplifier.

Linearization techniques include power back-off method, feed-forward method, negative feedback method, nonlinear device method (LINC), digital predistortion method and analog predistortion method[1,2,7]. These methods have been proposed to overcome nonlinear distortions. Among these traditional techniques, analog predistortion technique has the advantages of simple structure, higher stability, wider bandwidth, lower cost, so it has been widely used[3,6]. In this paper, a new type of analog predistortion is designed, first, this paper introduces analog predistortion technology and specific circuit, and then analysis the feasibility of the circuit from the aspects of theory, finally verify its performance through special electromagnetic simulation software ADS and compare with the experimental results.

\section{The analog predistortion technique}

The pre-distortion technique compensates the gain amplitude distortion (AM-AM) and phase distortion (AM-PM) of the power amplifier through a predistortion module that is inserted preceding the power amplifier. The design of predistortion linearization device using RF circuit is called analog predistortion technique. As shown in Fig.1, the transfer function of the predistortion block must be designed to have an exponential property to make transmission procedure remain linear. Since the analog predistortion circuit is simple, easy to implement, and the overall performance is good[2,3], it is widely used to improve the nonlinear distortion of the amplifier[4,7].The key to designing analog predistortion is to achieve the device with an actual circuit. 




Fig.1 Block diagram of the analog predistortion circuit

\section{Design of Analog predistortion linearizer}

The circuit is improved from the traditional parallel diode circuit, and the core device is a diode that generates nonlinear signals. The small signal equivalent circuit of the Schottky diode used is shown in Fig.2.

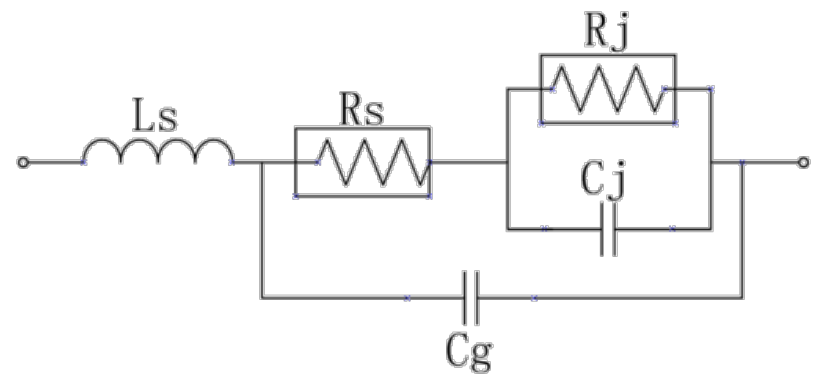

Fig.2 Small signal equivalent circuit diagram of the Schottky diode

The $I$ / V equation can be listed according to the current flowing through the Schottky diode, namely $I=I s(\exp a V-1)$ [3], where $\alpha=q / n k T, \alpha$ represents approximately $1 /(25 m V), q$ represents the charge of electrons, $k$ represents the boltzmann constant, $T$ represents the temperature, $n$ represents the idealized factor. The $I / V$ curve is shown in Fig.3. As shown in the figure, the resistance of diode is nonlinear, and it can produce many high-order harmonic components, this feature determines that it is suitable as a nonlinear signal generating part of the analog predistorter.

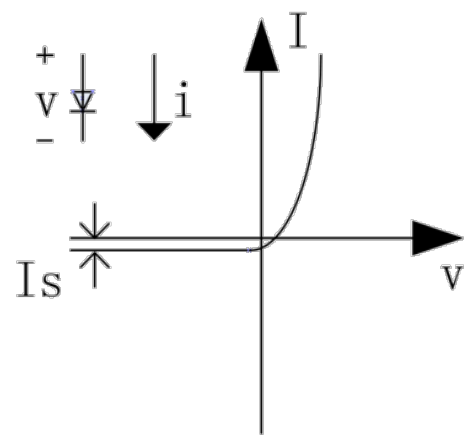

Fig.3 The I / V curve of the Schottky diode

\section{Software simulation and results}

The performance of the predistortion circuit is tested and verified by the special electromagnetic simulation software ADS2013. RT/duriod5880 is applied as the dielectric substrate.The Schottky diode is chosen from MA-COM company which is integrate with coplanar waveguides. The waveguide - microstrip transition structure is designed with HFSS, and the offset high resistance line is designed with ADS. The circuit diagram is shown in Fig.4. 


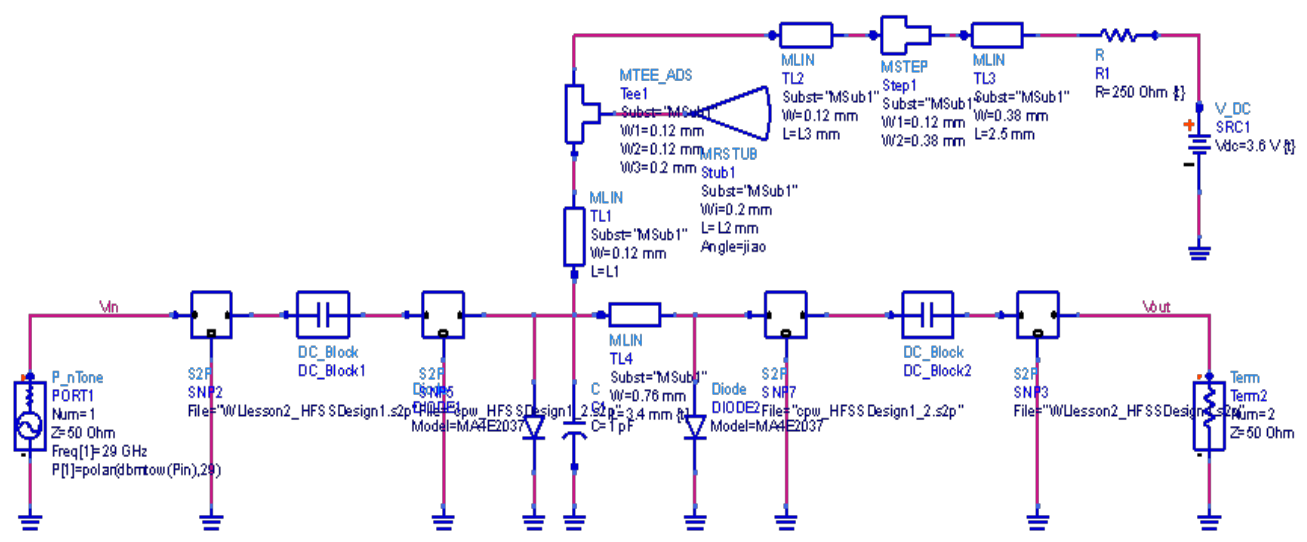

Fig.4 Diagram of analog predistortion circuit

The traditional predistortion circuit adopts closed-loop technology, which has poor stability, complex structure and high cost[3].In this paper, the traditional structure is improved, as shown in Fig.4, using open loop technology with the advantages of stability, higher bandwidth properties, this circuit can deal with the need of multi-carrier modulation signal, meantime, the circuit structure is simple because of less tuning parts, simple debug, and it is easy to integrate with less installation cost.

The circuit consists of two Schottky diodes in parallel with a capacitor, a bias resistor and a passive structure. The change of bias voltage and bias resistance can alter the current of the Schottky diode and change the shunt capacitance to adjust the nonlinear curve. The microstrip line length is $2.72 \mathrm{~mm}$, and its electrical length is $135^{\circ}$, capacitance initial value is $1 \mathrm{pF}$, resistance initial value of $300 \Omega$. The bias voltage, bias resistance and capacitance are scanned and the best results are shown in Fig. 3-2.
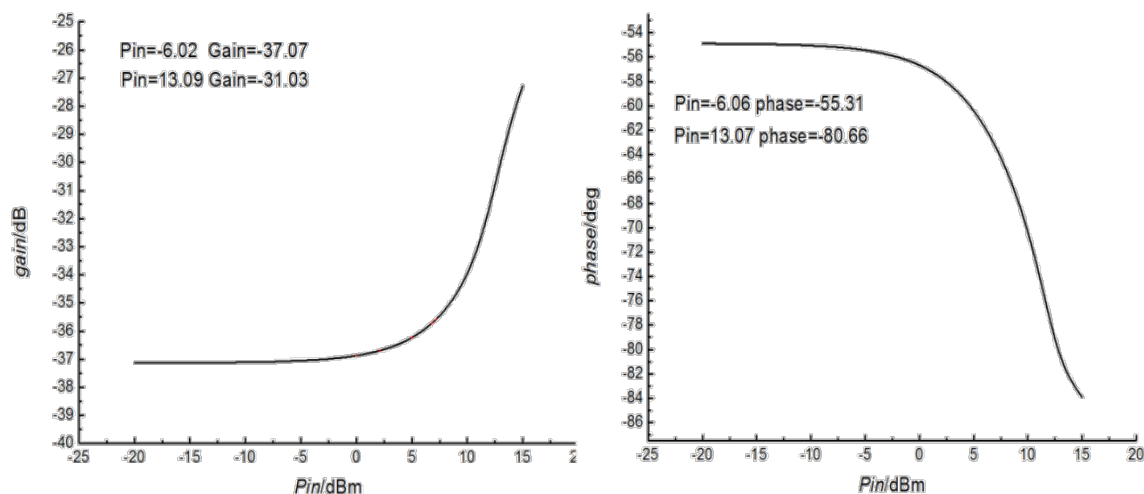

Fig.5 Simulation results diagram of predistortion circuit

As shown in Fig.5, bias voltage is set to $3.5 \mathrm{~V}$, bias resistors is set to $250 \Omega$, capacitors is set to $1 \mathrm{pF}$ while the input power is scanned from $-6 \mathrm{~dB}$ to $13 \mathrm{~dB}$, gain compensation attains $7 \mathrm{~dB}$, phase compensation attains $25^{\circ}$. It is easy to know that the smaller the current of the Schottky diode is, the weaker its nonlinear characteristics and the weaker the ability to generate the compensating signal. Increasing bias voltage and reducing bias resistance can both increase the current of Schottky diode. By changing the bias voltage or bias resistance, the demand for different compensation effects can be achieved. According to the value obtained by simulation, the object is assembled, and then the physical debugging is carried out by adjusting the bias voltage and bias resistance.

\section{Implementation and experimental results}

The predistortion linearizer is shown in Fig.6, and the input and output terminals use the waveguide coaxial adapter, and the amplitude compensation and phase compensation characteristics of the linearization device are tested with the vector network analyzer. By adjusting the bias voltage and bias resistance, the results are shown in Fig.7. Available in the input power for $-6 \mathrm{dBm} \sim 13 \mathrm{dBm}$ range, the amplitude of predistortion linearer compensation can reach $6.4 \mathrm{~dB}$, phase compensation can 
achieve $28^{\circ}$, and the nonlinear characteristics of the solid-state power amplifier reciprocal, can be used to improve solid-state power amplifier nonlinear distortion. Due to the inaccurate modeling of Schottky diode, there is a gap between the simulation results and the measured results. If a more accurate and practical spice parameter model can be established, the simulation and prediction of linearized circuit performance will play an important role.

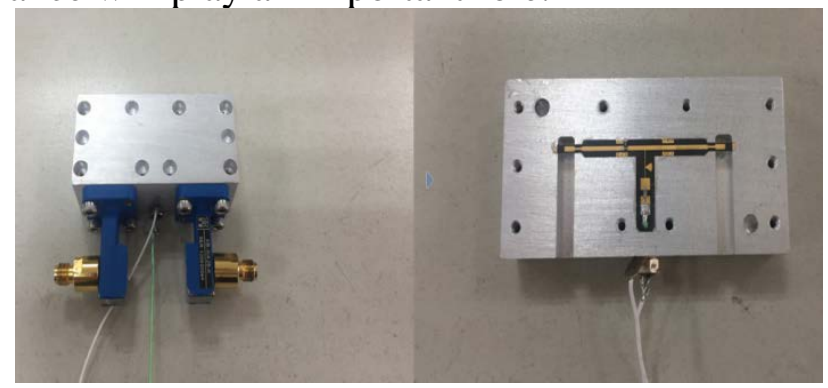

Fig.6 The predistortion linearization device

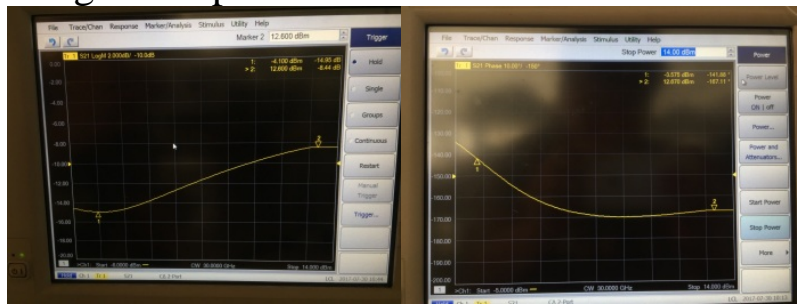

Fig.7 The test results of the predistortion linearization device

\section{Conclusion}

In this paper, two Schottky diodes are connected in parallel, by changing the bias voltage, bias resistors, the current of the Schottky diode is affected, the nonlinear characteristic of the diode is adjusted, and the linearization curve of different compensation degree is obtained. The vector network analyzer test results show that at the $30 \mathrm{GHz}$, gain amplitude compensation reaches $6.4 \mathrm{~dB}$, gain phase compensation reaches $28^{\circ}$. The circuit structure is simple and can be used in engineering practice.

\section{References}

[1] Li Dawei. Research on millimeter-wave predistortion linearization technology[D]. Chengdu: University of Electronic Science and Technology of China,2015.

[2] LI Dawei, XIE Xiaoqiang. A New Broadband Predistortion Structure for Millimeter-Wave Power Amplifier [J]. Journal Of Microwaves,2014(1):148-151.

[3] LI Shaolan, YAN Bo. Design of an Analog Pre-distortion Linearizer Using Schottky Diodes for Millimeter-Wave Power Amplifier [J]. Journal Of Microwaves,2012,28(1):70-72.

[4] ZHANG Yunhua, LI Dawei. A New Amplitude-phase Tunable Predistorter[J]. Electromechanical Components,2016(2):48-51.

[5] YAMAUCHI K, Mori K, NAKAYAMA M, et al. A microwave miniaturized linearizer using a parallel diode with a bias feed resistance [J]. Microwave Theory and Techniques, IEEE Transactions on, 1997, 45(12):2431-2435.

[6] CHUNG Y, DECKMAN B C, DELISIO M P. Linearization of a Spatially-Combined X-Band 100-W GaAs FET Power Amplifier System with Predistortion Linearizer[C].Compound Semiconductor Integrated Circuit Symposium (CSICS),2012 IEEE.

[7] YI J, YANG Y, PARK M., KANG W, and KIM B. Analog predistortion lineariser for high-power RF amplifiers[J]. Microwave Theory and Techniques, IEEE Trans,2000,48(12):2709-2713. 